\title{
Carvedilol improves liver cirrhosis in rats by inhibiting hepatic stellate cell activation, proliferation, invasion and collagen synthesis
}

\author{
LIPING LING $^{1}$, GUANGQI LI ${ }^{2}$, GUANGCHUAN WANG ${ }^{1}$, \\ DONGXIAO MENG ${ }^{1}$, ZHEN LI $^{1}$ and CHUNQING ZHANG ${ }^{1}$
}

${ }^{1}$ Department of Gastroenterology, Shandong Provincial Hospital Affiliated to Shandong University, Jinan, Shandong 250021; ${ }^{2}$ Department of Oncology, Binzhou People's Hospital, Binzhou, Shandong 256603, P.R. China

Received September 19, 2018; Accepted May 22, 2019

DOI: $10.3892 / \mathrm{mmr} .2019 .10401$

\begin{abstract}
Portal hypertension (PHT) is one of the most severe consequences of liver cirrhosis. Carvedilol is a first-line pharmacological treatment of PHT. However, the antifibrogenic effects of carvedilol on liver cirrhosis and the intrinsic mechanisms underlying these effects have not been thoroughly investigated. The present study aimed to investigate the antifibrogenic effects of carvedilol on liver cirrhosis in vivo and in vitro. Liver cirrhosis was induced in rats by carbon tetrachloride $\left(\mathrm{CCl}_{4}\right)$ administration for 9 weeks; carvedilol was administered simultaneously in the experimental group. Blood samples were collected for serum biochemistry. Liver tissues were used for fibrosis evaluation, histological examination, immunohistochemistry and western blot analysis. The human hepatic stellate cell (HSC) line LX-2 was used for in vitro studies. The effects of carvedilol on LX-2 cell proliferation and invasion were evaluated by Cell Counting Kit- 8 assay and Transwell invasion assays, respectively. The effect of carvedilol on transforming growth factor $\beta 1$ (TGF $\beta 1$ )-induced collagen synthesis in LX-2 cells and the molecular mechanisms were examined by western blot analysis. The results demonstrated that carvedilol improved $\mathrm{CCl}_{4}$-induced structural distortion and fibrosis in the liver. Carvedilol inhibited HSC activation, proliferation and invasion. Carvedilol inhibited HSC collagen synthesis through the TGF $\beta 1 / \mathrm{SMAD}$ pathway. In conclusion, carvedilol may alleviate liver cirrhosis in rats by inhibiting HSC activation, proliferation, invasion and collagen synthesis. Carvedilol may be a potential treatment of early-stage liver cirrhosis.
\end{abstract}

Correspondence to: Professor Chunqing Zhang, Department of Gastroenterology, Shandong Provincial Hospital Affiliated to Shandong University, 324 Jingwu Weiqi Road, Jinan, Shandong 250021, P.R. China

E-mail: zhangchunqing_sdu@163.com

Key words: carvedilol, hepatic stellate cell, liver cirrhosis, transforming growth factor $\beta 1$

\section{Introduction}

Portal hypertension (PHT) is one of the most severe clinical consequences of patients with liver cirrhosis, which results in life-threatening complications including variceal bleeding and hepatic encephalopathy (1). Previous studies have indicated that increased intrahepatic vascular resistance (IHVR) is the initial and determinant factor of PHT (1-3). Various structural and functional factors are responsible for the IHVR increase $(2,4-6)$. The formation of fibrous septa and regenerative nodules causes the distortion and compression of the venous system, which increases the resistance to portal venous blood flow $(1,2,6)$. The progressive process of cirrhosis is characterized by the excessive deposition of extracellular matrix (ECM) proteins including type I collagen (Col I), Col III and fibronectin (FN). Hepatic stellate cells (HSCs) are the primary cells responsible for liver cirrhosis (7). Upon liver injury, quiescent HSCs acquire an activated phenotype, migrate to the damaged region, proliferate and produce ECM proteins (8-10). Transforming growth factor $\beta 1$ (TGF $\beta 1$ ), which is the most potent profibrogenic cytokine in the liver, promotes the synthesis of ECM proteins in HSCs $(11,12)$. Multiple signaling pathways, including TGF/1/SMAD, PI3K/AKT and ERK, are involved in TGF $\beta 1$ signal transduction.

Carvedilol, a novel non-selective $\beta$-blocker (NSBB), is an antagonist of non-selective $\beta$ - and selective $\alpha_{1}$-adrenoreceptors that effectively reduces portal pressure (13). Studies of the role of carvedilol in the reduction of portal pressure mainly focus on hemodynamics. Previous studies have reported that carvedilol improves myocardial and renal fibrosis $(14,15)$. A small number of studies have examined the antifibrogenic effect of carvedilol on liver cirrhosis, and the underlying mechanisms are not well described (16). In chronic liver disease, portal pressure is mainly determined by the severity of the destruction of hepatic architecture (17). Therefore, it was hypothesized that the antifibrogenic effect of carvedilol may be involved in reducing the portal pressure. The present study aimed to investigate the antifibrogenic effect of carvedilol in vivo and in vitro. The rat model of liver cirrhosis induced by carbon tetrachloride was used to investigate the antifibrogenic effect of carvedilol in vivo. In vitro, the human line LX-2 was used to explore the mechanisms underlying carvedilol function. 


\section{Materials and methods}

Materials. Carbon tetrachloride $\left(\mathrm{CCl}_{4}\right)$ was purchased from Sinopharm Chemical Reagent Co., Ltd. Carvedilol used in animal experiments was obtained from Qilu Pharmaceutical Co., Ltd. Carvedilol used in the cell-based experiments was purchased from Sigma-Aldrich (Merck KGaA). Recombinant human TGF $\beta 1$ was obtained from PeproTech, Inc. Specific inhibitor of SMAD3 (SIS3) was purchased from Medchem Express.

Animals. A total of 40 male Wistar rats (weight, 180-200 g; age, 8 weeks) were purchased from the Central Animal Care Facility of Shandong University (Jinan, China). The rats were housed in the animal care facility under temperatureand humidity-controlled conditions (temperature, $22-24^{\circ} \mathrm{C}$; humidity, 50 \pm ) with a 12 -h light-dark cycle and were provided free access to food and water. The mortality rate was $20 \%$, and 13 rats were sacrificed for histological evaluation of liver cirrhosis and measurement of portal pressure. All rats were sacrificed under anesthesia induced by intraperitoneal injection of pentobarbital $(30 \mathrm{mg} / \mathrm{kg})$. All animal experiments were carried out in accordance with the Guide for the Care and Use of Laboratory Animals. All procedures were approved by the Animal Care and Use Committee of Shandong Provincial Hospital affiliated to Shandong University (approval no. 2018-005).

Induction of liver cirrhosis by $\mathrm{CCl}_{4}$ and administration of carvedilol. Cirrhosis was induced by the intraperitoneal (i.p.) injection of $\mathrm{CCl}_{4}$, as previously described (18). Rats were randomly divided into three groups: i) Control, which received an i.p. injection of olive oil $(0.5 \mathrm{ml} / \mathrm{kg}$ body weight) twice weekly for 9 weeks; ii) $\mathrm{CCl}_{4}$-intoxicated, which received an i.p. injection of $\mathrm{CCl}_{4}\left(1 \mathrm{ml} / \mathrm{kg} ; \mathrm{CCl}_{4}\right.$ to olive oil v/v ratio, 1:1) twice weekly for 9 weeks; and iii) $\mathrm{CCl}_{4}+$ carvedilol-treated, which received an i.p. injection of $\mathrm{CCl}_{4}\left(1 \mathrm{ml} / \mathrm{kg} ; \mathrm{CCl}_{4}\right.$ to olive oil $\mathrm{v} / \mathrm{v}$ ratio, 1:1) twice weekly for 9 weeks as well as concurrent treatment with carvedilol $(10 \mathrm{mg} / \mathrm{kg})$ via gavage daily for 9 weeks. Rats in the control and $\mathrm{CCl}_{4}$-intoxicated groups received the vehicle ( $2 \mathrm{ml}$ saline) by gavage daily for 9 weeks.

Serum assays. At the end of the experiment, rats were weighed and anesthetized. Laparotomy was performed to expose the inferior vena cava. In total, $3 \mathrm{ml}$ venous blood was collected from each rat into procoagulant vacuum tubes from the inferior vena cava and centrifuged at 3,000 $\mathrm{x} g$ for $10 \mathrm{~min}$ at $4^{\circ} \mathrm{C}$. Supernatant was collected and stored at $-80^{\circ} \mathrm{C}$ until biochemical assays were performed. Serum aspartate aminotransferase (AST), alanine aminotransferase (ALT) and albumin (ALB) levels were measured using an AU1000 fully automatic biochemical analyzer (Olympus Corporation).

Histological examination. Following blood sample collection, rats were sacrificed and livers were harvested. Liver specimens were fixed in $4 \%$ paraformaldehyde for $24 \mathrm{~h}$ at room temperature, embedded in paraffin and cut into $4-\mu$ m-thick sections. Sections were stained with hematoxylin and eosin (H\&E) for $5 \mathrm{~min}$ and for $30 \mathrm{sec}$, respectively, at room temperature for histopathological examination, and photographed under an
Olympus BX63F light microscope (Olympus Corporation; magnification, $x 100)$. Sections were stained with sirius red (S-R) dye for $1 \mathrm{~h}$ at room temperature and with hematoxylin for $3 \mathrm{~min}$ at room temperature in order to visualize collagen deposition; the sections were photographed under a light microscope and under a Nikon Eclipse Ci-E polarized light microscope (Nikon Corporation; magnification, x200). The picrosirius-polarization method was used to evaluate the distribution of Col I (thick, strongly birefringent, yellow or red fibers) and Col III (thin, weakly birefringent, green fibers), as previously described (19). The collagen-positive area to total area ratio was quantified using Image-Pro Plus 6.0 (Media Cybernetics, Inc.).

Immunohistochemical analysis. Immunohistochemistry was performed using a Polink-2 Plus Polymer-Horseradish Peroxidase (HRP) Anti-Rabbit Immunoglobulin G (IgG) Detection System (Beijing Zhongshan Golden Bridge Biotechnology Co., Ltd.), according to the manufacturer's protocol. Liver sections were deparaffinized and rehydrated in descending series of ethanol. After heat-mediated antigen retrieval with citrate buffer at $120^{\circ} \mathrm{C}$ for $3 \mathrm{~min}$, the sections were incubated with $3 \%$ hydrogen peroxide for $10 \mathrm{~min}$ at room temperature to suppress endogenous peroxidase activity. The sections were incubated with polyclonal rabbit anti-rat $\alpha$-smooth muscle actin ( $\alpha$-SMA; 1:300; cat. no. A03744; Boster Biological Technology) at $4^{\circ} \mathrm{C}$ overnight, and were subsequently warmed at $37^{\circ} \mathrm{C}$ for $30 \mathrm{~min}$. Following incubation with the goat anti-rabbit IgG HRP-conjugated secondary antibody included in the kit at $37^{\circ} \mathrm{C}$ for $30 \mathrm{~min}$, the sections were stained with diaminobenzidine solution, counterstained with hematoxylin and dehydrated through an increasing gradient of ethanol, according to the manufacturer's protocol. Images were captured under an Olympus BX63F light microscope. Sections incubated with PBS instead of the primary antibody were used as negative controls.

Western blotting. Liver tissue samples were stored in liquid nitrogen. Proteins were extracted from cells and liver tissues using the Tissue or Cell Total Protein Extraction kit (Sangon Biotech Co., Ltd.) according to the manufacturer's protocol. Protein concentrations were measured using a Bicinchoninic Acid Protein assay kit (Beyotime Institute of Biotechnology). Equal amounts $(50 \mu \mathrm{g} / \mathrm{well})$ of proteins were separated by $8 \%$ SDS-PAGE and transferred to PVDF membranes (EMD Millipore). Following blocking in 5\% skimmed milk for $1 \mathrm{~h}$ at room temperature, the membranes were incubated with the primary antibodies at $4^{\circ} \mathrm{C}$ overnight, followed by incubation with the HRP-conjugated secondary antibodies goat anti-rabbit (Beijing Zhongshan Golden Bridge Biotechnology Co., Ltd.; cat. no. ZB-5301; 1:5,000) or rabbit anti-goat (Beijing Zhongshan Golden Bridge Biotechnology Co., Ltd.; cat. no. ZB-2306; 1:5,000) for $2 \mathrm{~h}$ at room temperature. The signals of the target proteins were detected by enhanced chemiluminescence using Amersham Imager 600 (GE Healthcare). ImageJ software (version 1.46r; National Institutes of Health, Bethesda, MD, USA) was used to perform densitometric analysis. Band intensities were normalized to GAPDH. The primary antibodies used were as follows: Monoclonal rabbit anti-total AKT (cat. no. 4691; 1:1,000), 
Table I. Effects of CARV on body weight and biochemical parameters in $\mathrm{CCl}_{4}$-intoxicated cirrhotic rats.

\begin{tabular}{lccc}
\hline Parameter & Control $(\mathrm{n}=7)$ & $\mathrm{CCl}_{4}$-intoxicated $(\mathrm{n}=6)$ & $\mathrm{CCl})_{4}+\mathrm{CARV}(\mathrm{n}=6)$ \\
\hline Bodyweight $(\mathrm{g})$ & $434.3 \pm 21.9$ & $380.0 \pm 25.8^{\mathrm{a}}$ & $341.8 \pm 40.5^{\mathrm{b}}$ \\
ALT (U/l) & $50.98 \pm 12.76$ & $99.04 \pm 14.04^{\mathrm{a}}$ & $79.76 \pm 11.68^{\mathrm{b}}$ \\
AST (U/l) & $90.45 \pm 12.87$ & $170.45 \pm 42.50^{\mathrm{a}}$ & $118.65 \pm 21.70^{\mathrm{c}}$ \\
ALB (g/l) & $27.63 \pm 2.89$ & $22.53 \pm 1.20^{\mathrm{a}}$ & $21.20 \pm 1.84$
\end{tabular}

${ }^{\mathrm{a}} \mathrm{P}<0.01$ vs. control. ${ }^{\mathrm{b}} \mathrm{P}<0.05$ vs. $\mathrm{CCl}_{4} .{ }^{\mathrm{c}} \mathrm{P}<0.01$ vs. $\mathrm{CCl}_{4}$. ALT, alanine aminotransferase; ALB, albumin; AST, aspartate aminotransferase; CARV, carvedilol; $\mathrm{CCl}_{4}$, carbon tetrachloride.

monoclonal rabbit anti-phosphorylated (p)-AKT (cat. no. 4060; 1:1,000), polyclonal rabbit anti-total-p44/42 MAPK (t-ERK1/2; cat. no. 9102; 1:1,000), polyclonal rabbit anti-phospho-p44/42 MAPK (p-ERK1/2; cat. no. 9101; 1:1,000), monoclonal rabbit anti-total SMAD3 (cat. no. 9523; 1:1,000), monoclonal rabbit anti-p-SMAD2 (cat. no. 3108; 1:1,000) and monoclonal rabbit anti-GAPDH (cat. no. 5174; 1:1,000) purchased from Cell Signaling Technology, Inc.; polyclonal rabbit anti-FN (cat. no. ab2413; 1:1,000), monoclonal rabbit anti- $\alpha$-SMA (cat. no. ab32575; 1:1,000), monoclonal rabbit anti-total SMAD2 (cat. no. ab40855; 1:2,000) and monoclonal rabbit anti-p-SMAD3 (cat. no. ab52903; 1:2,000) purchased from Abcam; and monoclonal goat anti-Col I (cat. no. 1310-01; 1:1,000) purchased from SouthernBiotech (Birmingham, AL, USA).

Cell culture. LX-2 is an activated human HSC cell line that is widely used as a model for hepatic fibrosis (9). LX-2 cells were a gift from Professor Weifen Xie (Shanghai Changzheng Hospital, The Second Military Medical University, Shanghai, China). Cells were cultured in DMEM (Gibco; Thermo Fisher Scientific, Inc., Waltham, MA, USA) supplemented with $2 \%$ fetal bovine serum (FBS; Gibco; Thermo Fisher Scientific, Inc.), $100 \mathrm{U} / \mathrm{ml}$ of penicillin and $100 \mu \mathrm{g} / \mathrm{ml}$ of streptomycin (Gibco; Thermo Fisher Scientific, Inc.) at $37^{\circ} \mathrm{C}$ in a humidified incubator with $5 \% \mathrm{CO}_{2}$.

Cell Counting Kit-8 (CCK-8). LX-2 cells were plated in 96-well culture plates $\left(5 \times 10^{3}\right.$ cells/well) in triplicate and incubated at $37^{\circ} \mathrm{C}$ overnight. Subsequently, the cells were incubated with $0,1,2,5$ or $10 \mu \mathrm{M}$ carvedilol in a humidified incubator with $5 \% \mathrm{CO}_{2}$ at $37^{\circ} \mathrm{C}$ for $24 \mathrm{~h}$. Following the treatment, $10 \mu \mathrm{l} \mathrm{CCK}-8$ solution (Dojindo Molecular Technologies, Inc.) was added to each well. The plates were incubated in a humidified incubator with $5 \% \mathrm{CO}_{2}$ at $37^{\circ} \mathrm{C}$ for $2 \mathrm{~h}$. Cell viability was calculated according to the manufacturer's protocol (Dojindo Molecular Technologies, Inc.). Optical density was measured at $450 \mathrm{~nm}$ using a spectrophotometer (Thermo Fisher Scientific, Inc.). The experiment was repeated three times.

Transwell invasion assay. Serum-starved LX-2 cells (1x10 $/ \mathrm{ml})$ treated with $0,1,2,5$ or $10 \mu \mathrm{M}$ carvedilol in $100 \mu \mathrm{l}$ serum-free culture medium were seeded into the upper chamber of a 24-well Transwell plate, and the membranes of the upper chamber were coated with Matrigel (BD Biosciences). Culture medium with $10 \%$ FBS was added to the lower chamber.
Serum-free culture medium was used in the lower chamber as a non-induced control. Cells were incubated in a humidified incubator with $5 \% \mathrm{CO}_{2}$ at $37^{\circ} \mathrm{C}$ for $24 \mathrm{~h}$. Subsequently, the cell medium was discarded, and cells in the upper chamber were removed by a cotton swab. The cells on the lower surface of the chamber were fixed with $4 \%$ paraformaldehyde for $20 \mathrm{~min}$ at room temperature and stained with hematoxylin for $10 \mathrm{~min}$ at room temperature. The migrated cells were assessed in six randomly selected fields under an Olympus BX63F light microscope.

Statistical analysis. All data are presented as the mean \pm SD. SPSS Statistics 20.0 (IBM Corp.) was used for statistical analyses. Comparisons were performed using one-way ANOVA followed by Dunnett's test. $\mathrm{P}<0.05$ was considered to indicate a statistically significant difference.

\section{Results}

Effects of carvedilol on body weight and biochemical parameters of $\mathrm{CCl}_{4}$-intoxicated rats. The body weight of the $\mathrm{CCl}_{4}$-intoxicated group was significantly lower compared with the control group $(\mathrm{P}<0.01$; Table $\mathrm{I})$. The body weight of the $\mathrm{CCl}_{4}+$ carvedilol-treated group was lower compared with the $\mathrm{CCl}_{4}$-intoxicated group $(\mathrm{P}<0.05$; Table I). Compared with the control group, $\mathrm{CCl}_{4}$ treatment significantly increased the serum levels of ALT and AST $(\mathrm{P}<0.01)$, whereas co-treatment with carvedilol decreased the elevated levels of ALT and AST $(\mathrm{P}<0.05$ and $\mathrm{P}<0.01$, respectively; Table I). Compared with the control group, $\mathrm{CCl}_{4}$ significantly decreased the serum level of ALB ( $\mathrm{P}<0.01$; Table I); however, not significant difference was identified in the levels of ALB between the $\mathrm{CCl}_{4}$-intoxicated and $\mathrm{CCl}_{4}+$ carvedilol-treated groups.

Carvedilol improves $\mathrm{CCl}_{4}$-induced structural distortion and fibrosis in the liver. Histological differences among the groups were demonstrated by H\&E staining of liver sections. Liver sections of the control group exhibited normal lobular architecture, whereas liver sections of the $\mathrm{CCl}_{4}$-intoxicated group exhibited typical architectural distortions with regenerative nodules surrounded by proliferative connective tissue; co-treatment with carvedilol notably improved the architectural destruction induced by $\mathrm{CCl}_{4}$ (Fig. 1A). S-R staining revealed that, compared with control group rats, $\mathrm{CCl}_{4}$ treatment resulted in excessive collagen deposition in cirrhotic livers, which was notably reduced by co-treatment with 

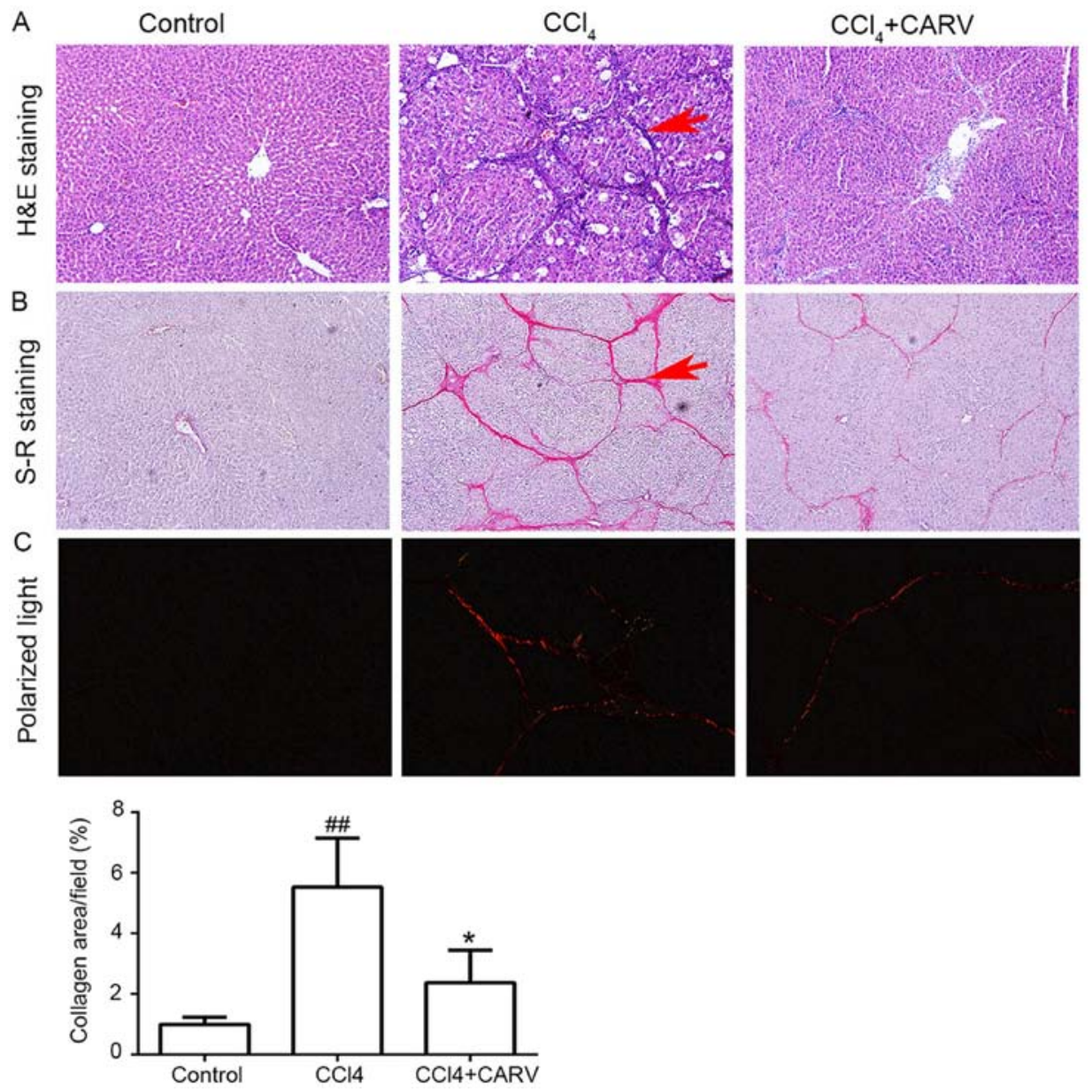

Figure 1. Carvedilol improves $\mathrm{CCl}_{4}$-induced structural distortion and fibrosis. (A) Representative histological images of H\&E stained liver sections of non-cirrhotic Control rats, $\mathrm{CCl}_{4}$-intoxicatd rats and $\mathrm{CCl}_{4}$-intoxicatd rats treated with carvedilol; magnification, $\mathrm{x} 100$. Arrow indicates the regenerative nodule. (B) Images of S-R staining of livers from rats in each group; magnification, x100. Arrow indicates the collagen deposition. (C) Quantification of collagen-positive area in liver sections by polarized light microscopy; magnification, $\mathrm{x} 200$. ${ }^{\# \#} \mathrm{P}<0.01$ vs. control group; ${ }^{*} \mathrm{P}<0.05$ vs. $\mathrm{CCl} \mathrm{l}_{4}$-intoxicated group. CARV, carvedilol; $\mathrm{CCl}_{4}$, carbon tetrachloride; $\mathrm{H} \& \mathrm{E}$, hematoxylin and eosin; $\mathrm{S}-\mathrm{R}$, sirius red.

carvedilol (Fig. 1B). Collagen deposition was quantified by the picrosirius-polarization method. The collagen-positive area to total area ratio in the $\mathrm{CCl}_{4}$-intoxicated group was significantly higher compared with the control group $(\mathrm{P}<0.01$; Fig. 1C), whereas co-treatment with carvedilol significantly lowered the $\mathrm{CCl}_{4}$-induced collagen accumulation $(\mathrm{P}<0.05)$.

Carvedilol inhibits HSC activation in vivo. $\alpha$-SMA is a marker of HSC activation (8). Immunohistochemical assay results demonstrated that the expression of $\alpha$-SMA was notably higher in liver tissues of the $\mathrm{CCl}_{4}$-intoxicated group compared with the control group, whereas co-treatment with carvedilol suppressed the $\mathrm{CCl}_{4}$-induced increase of $\alpha$-SMA (Fig. 2A). Compared with the control group, the protein expression of $\alpha$-SMA was significantly upregulated by $\mathrm{CCl}_{4}(\mathrm{P}<0.01$; Fig. 2B), determined by western blotting, and co-treatment with carvedilol decreased the upregulated $\alpha$-SMA protein expression levels $(\mathrm{P}<0.01)$. These results demonstrated that carvedilol may have an inhibitory effect on HSC activation in cirrhotic livers of rats.

Carvedilol inhibits HSC activation in vitro. LX-2 cells exhibit an activated HSC phenotype, which was assessed as previously described (9). LX-2 cells were treated with carvedilol at concentrations of $0,1,2,5$ and $10 \mu \mathrm{M}$ for $24 \mathrm{~h}$. $\alpha$-SMA protein expression in LX-2 cells was significantly reduced by carvedilol at 5 and $10 \mu \mathrm{M}$ compared with untreated control cells $(\mathrm{P}<0.01$; Fig. $2 \mathrm{C})$. This result further demonstrated that carvedilol may have an inhibitory effect on HSCs activation in in vitro.

Carvedilol inhibits HSC proliferation. LX-2 cells were treated with carvedilol at concentrations ranging from 1 to $80 \mu \mathrm{M}$ for $24 \mathrm{~h}$. Carvedilol inhibited the proliferation of LX-2 cells in a dose-dependent manner (Fig. 3A). Carvedilol concentrations of $\leq 10 \mu \mathrm{M}$ were selected for subsequent cell experiments as carvedilol did not significantly reduce cell viability at this concentration.

Carvedilol decreases the invasive ability of HSCs. The number of migrated FBS-induced LX-2 cells was significantly higher compared with the non-induced control group $(\mathrm{P}<001$; Fig. 3B), whereas FBS-induced migration was significantly inhibited by carvedilol at concentrations of 5 and $10 \mu \mathrm{M}$ $(\mathrm{P}<0.05$ and $\mathrm{P}<0.01$, respectively). This result demonstrated that carvedilol may inhibit the invasive ability of HSCs. 


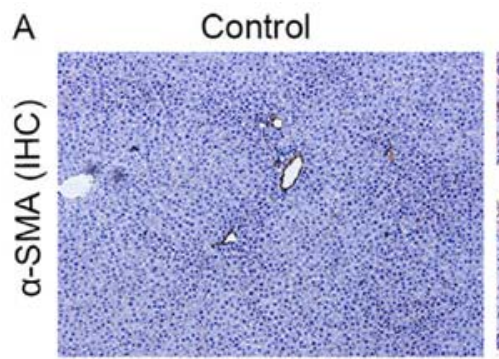

B

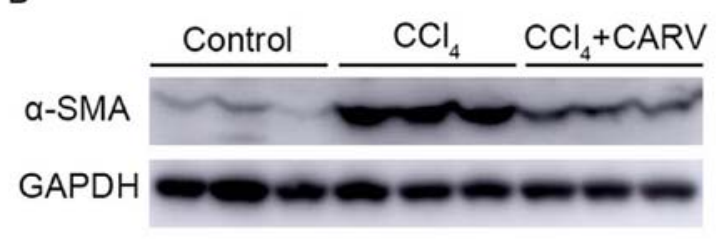

C

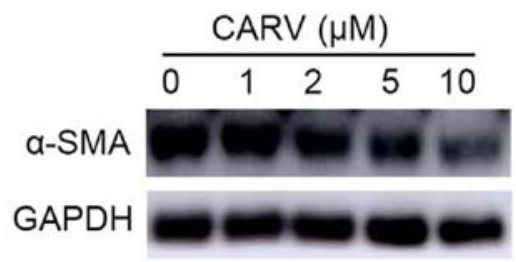

$\mathrm{CCl}_{4}$
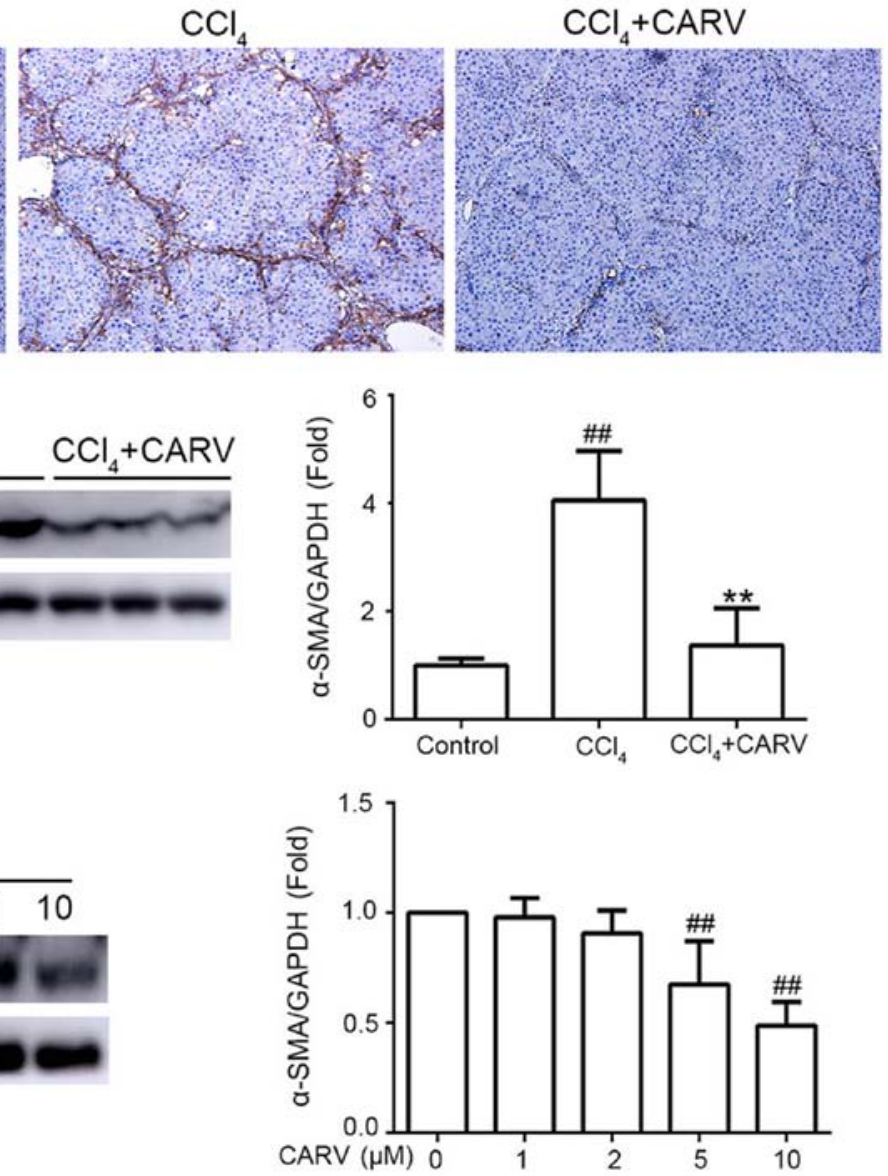

Figure 2. Carvedilol inhibits hepatic stellate cell activation in vivo and in vitro. (A) Representative images of immunohistochemical staining of $\alpha$-SMA of liver sections of non-cirrhotic Control rats, $\mathrm{CCl}_{4}$-intoxicatd rats and $\mathrm{CCl}_{4}$-intoxicatd rats treated with carvedilol; magnification, $\mathrm{x} 100$. (B) Protein expression levels of $\alpha$-SMA in liver tissues were quantified by western blotting and densitometric analysis. (C) $\alpha$-SMA protein expression levels in LX-2 cells treated with various concentrations of carvedilol. ${ }^{\# \#} \mathrm{P}<0.01$ vs. control; ${ }^{* *} \mathrm{P}<0.01$ vs. $\mathrm{CCl}_{4}$-intoxicated group. $\alpha$-SMA, $\alpha$-smooth muscle actin; $\mathrm{CARV}$, carvedilol; $\mathrm{CCl}$, carbon tetrachloride.
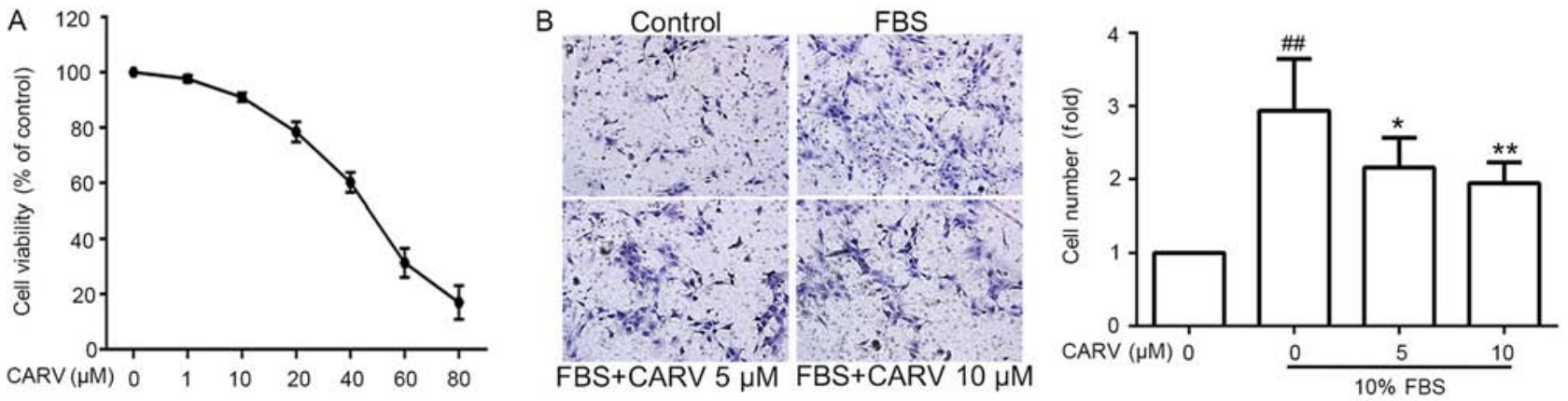

Figure 3. Carvedilol inhibits proliferative and invasive abilities of hepatic stellate cells. (A) Effects of a range of carvedilol concentrations on the proliferation of LX-2 cells were evaluated by Cell Counting Kit-8 assay. (B) Effects of carvedilol on the invasive ability of LX-2 cells were examined by Transwell migration assay; magnification, $x 100 .{ }^{\# \#} \mathrm{P}<0.01$ vs. serum-free Control; ${ }^{*} \mathrm{P}<0.05$ vs. $0 \mu \mathrm{M}$ carvedilol $+10 \%$ FBS; ${ }^{* *} \mathrm{P}<0.01$ vs. $0 \mu \mathrm{M}$ carvedilol $+10 \%$ FBS. CARV, carvedilol.

Carvedilol inhibits TGFß1-induced collagen synthesis of HSCs. LX-2 cells were seeded in 6-well plates and incubated overnight. The cells were stimulated with TGF $\beta 1$ at concentrations of $10,20,30$ and $50 \mathrm{ng} / \mathrm{ml}$ for $24 \mathrm{~h}$. Compared with the control group, stimulation with TGF $\beta 1$ significantly increased the protein expression levels of $\mathrm{Col}$ I and $\mathrm{FN}(\mathrm{P}<0.05$ and $\mathrm{P}<0.01$, respectively; Fig. 4A). TGF $\beta 1(20 \mathrm{ng} / \mathrm{ml})$ was used to stimulate LX-2 cells for $24 \mathrm{~h}$ with or without co-treatment with carvedilol. The TGF $\beta 1$-induced upregulation of Col I and FN was downregulated by carvedilol at 5 and $10 \mu \mathrm{M}(\mathrm{P}<0.05$ and $\mathrm{P}<0.01$, respectively; Fig. 4B).

Carvedilol inhibits TGFB1-induced HSC collagen synthesis via the TGF $\beta 1 / S M A D$ pathway. Signaling molecules 
A
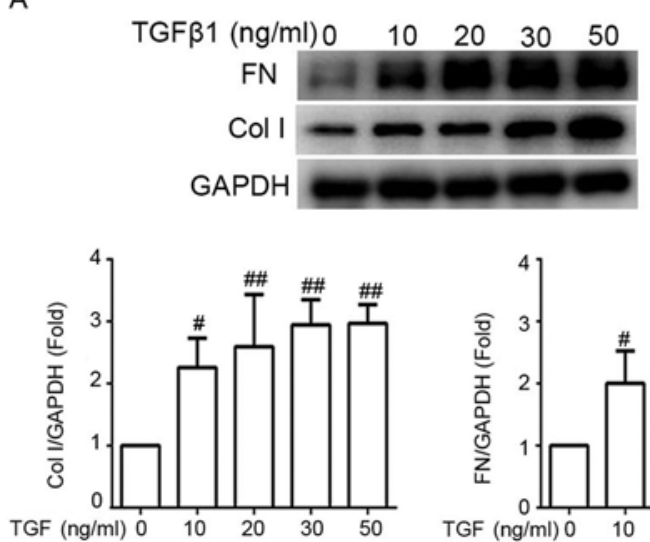

B

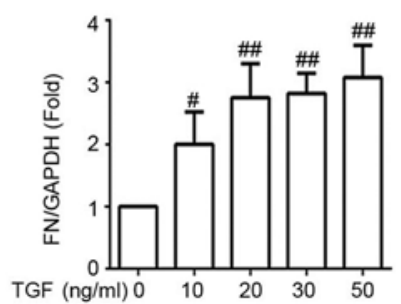

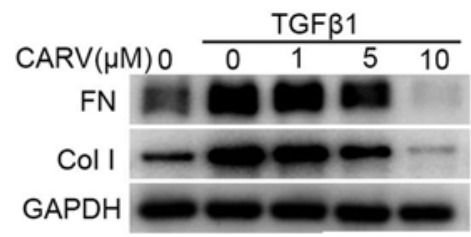

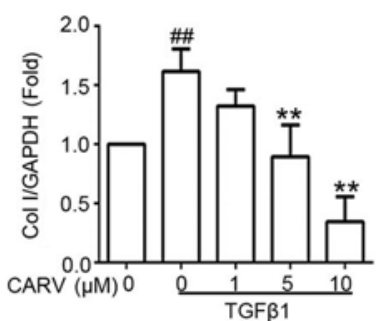

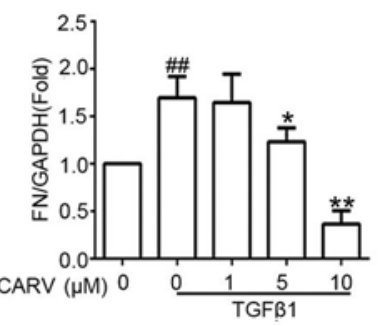

Figure 4. Carvedilol inhibits TGF $\beta 1$-induced collagen synthesis of hepatic stellate cells. (A) TGF $\beta 1$-induced Col I and FN protein expression in LX-2 cells. (B) Carvedilol inhibited TGF $\beta 1$-induced Col I and FN protein expression in LX-2 cells. " $\mathrm{P}<0.05$ vs. control; ${ }^{\# \#} \mathrm{P}<0.01$ vs. control; ${ }^{*} \mathrm{P}<0.05$ vs. $0 \mu \mathrm{M}$ carvedilol + TGF $\beta 1 ;{ }^{* *} \mathrm{P}<0.01$ vs. $0 \mu \mathrm{M}$ carvedilol + TGF $\beta 1$. CARV, carvedilol; Col I, type I collagen; FN, fibronectin; TGF $\beta 1$, transforming growth factor $\beta 1$.
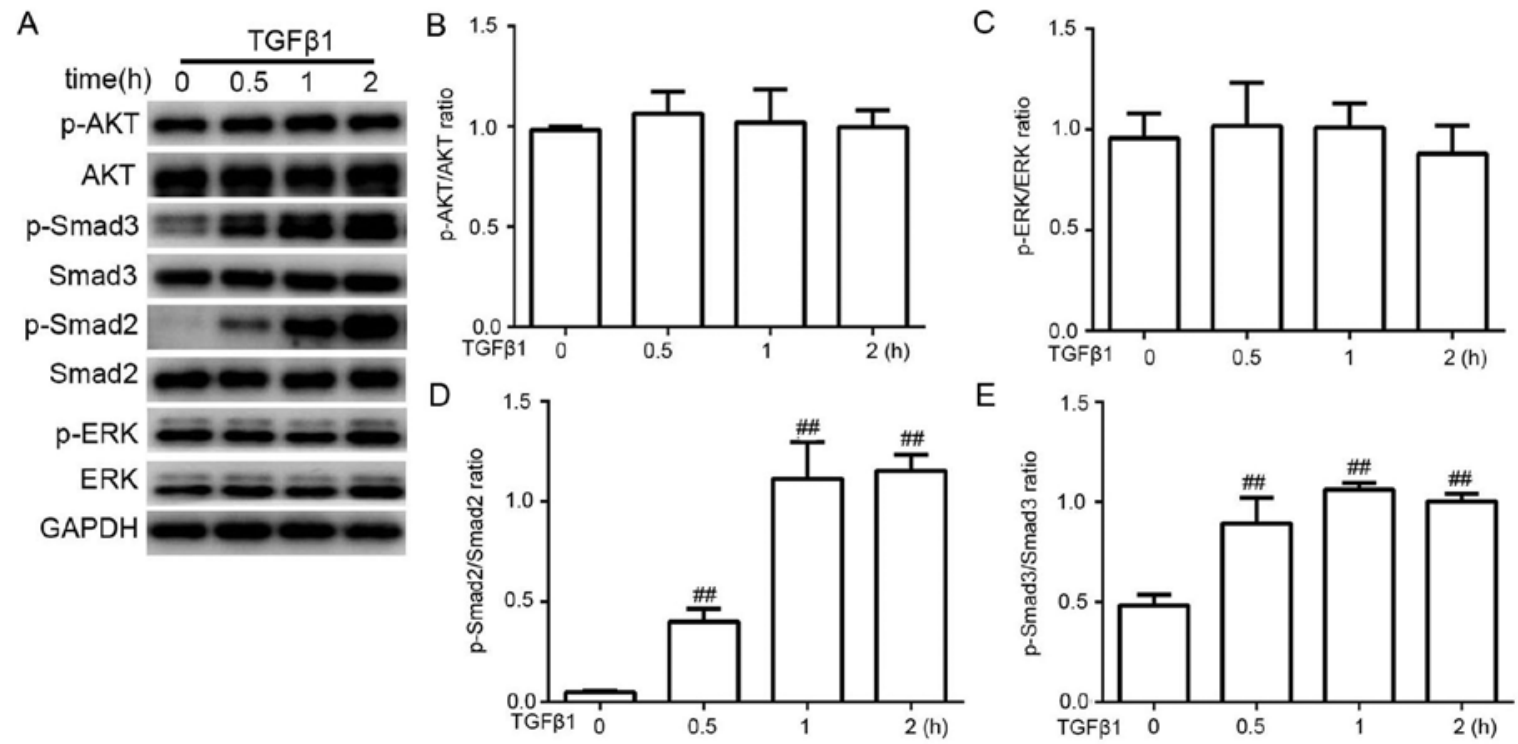

Figure 5. AKT, ERK, SMAD2 and SMAD3 phosphorylation levels in TGF 31 -stimulated LX-2 cells. (A) Representative western blotting analysis of protein expressions and densitometric analysis of the phosphorylation levels of (B) AKT, (C) ERK, (D) SMAD2 and (E) SMAD3 following TGF 31 stimulation. ${ }^{\# \#} \mathrm{P}<0.01$ vs. control. AKT, protein kinase B; $\mathrm{p}$, phosphorylated; TGF $\beta 1$, transforming growth factor $\beta 1$.

downstream of TGF $\beta 1$ were screened in LX-2 cells treated with TGF $\beta 1(20 \mathrm{ng} / \mathrm{ml})$ for $0.5,1$ and $2 \mathrm{~h}$ (Fig. 5). AKT and ERK phosphorylation levels did not change significantly (Fig. 5B and C, respectively), whereas the expression levels of p-SMAD2 and p-SMAD3 were upregulated in LX-2 cells stimulated with TGF $\beta 1$ compared with the untreated control $(\mathrm{P}<0.0$; Fig. 5D and E, respectively).

TGF 11 was used to stimulate LX-2 cells for $0.5 \mathrm{~h}$ with or without co-treatment with carvedilol. The phosphorylation of SMAD2 and SMAD3 induced by TGF $\beta 1$ was suppressed by pretreatment with carvedilol at concentrations of 5 and $10 \mu \mathrm{M}$ $(\mathrm{P}<0.05$ and $\mathrm{P}<0.01$, respectively; Fig. 6A and $\mathrm{B})$. SIS3 is a potent and selective inhibitor of SMAD3 (20). TGF $\beta 1$ was used to stimulate LX-2 cells for $24 \mathrm{~h}$ with or without co-treatment with $10 \mu \mathrm{M}$ SIS3. Pretreatment with SIS3 significantly decreased the TGF $\beta 1$-induced upregulation of Col I and FN $(\mathrm{P}<0.01$; Fig. 6C).

\section{Discussion}

Recent advances in our understanding of the pathophysiology of PHT and liver cirrhosis has resulted in improved management for patients with cirrhosis (2). As a novel NSBB, carvedilol reduces portal pressure more effectively than traditional NSBBs, such as propranolol and nadolol (21). For patients with compensated cirrhosis, the goal of treatment is to delay the development of liver cirrhosis and PHT. Therefore, the effects of carvedilol on liver cirrhosis were explored in vivo and in vitro to discover the potential role of carvedilol in treating early-stage liver cirrhosis.

Previous studies have reported that carvedilol has antioxidant, anti-proliferative, anti-inflammatory, anti-angiogenic and antifibrogenic effects $(14,15,22-24)$. Hamdy et al demonstrated that carvedilol had potent antifibrotic effects in chronic $\mathrm{CCl}_{4}$-induced liver damage, but the underlying mechanisms 
A
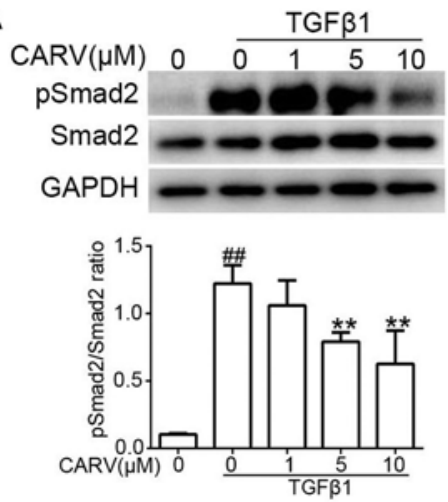

B
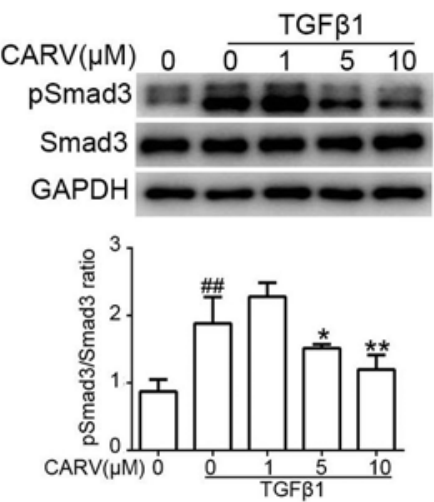

C
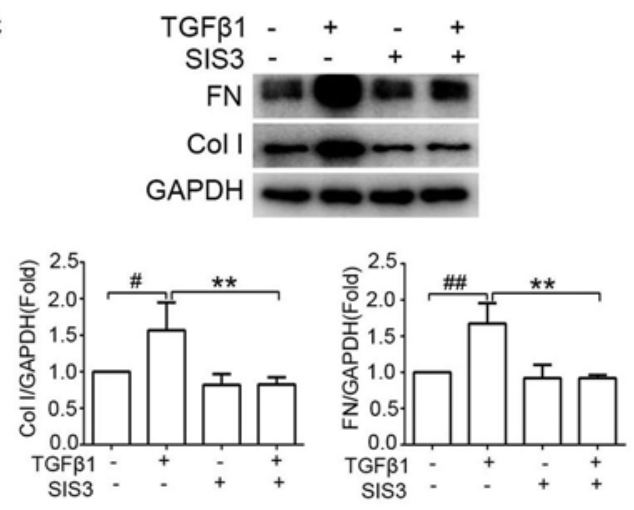

Figure 6. Carvedilol inhibits TGF $\beta 1$-induced hepatic stellate cell collagen synthesis through the TGF $\beta / S M A D$ pathway. (A) Carvedilol suppressed the TGF $\beta 1$-induced upregulation of p-SMAD2. (B) Carvedilol attenuated the TGF 31 -induced upregulation of p-SMAD3. (C) SIS3 treatment decreased the TGF $\beta 1$-induced expression of Col I and FN. ${ }^{.} \mathrm{P}<0.05$ vs. control; ${ }^{\# \#} \mathrm{P}<0.01$ vs. control; ${ }^{*} \mathrm{P}<0.05$ vs. $0 \mu \mathrm{M}$ carvedilol + TGF $\beta 1,{ }^{* *} \mathrm{P}<0.01$ vs. $0 \mu \mathrm{M}$ carvedilol + TGF $\beta 1$. CARV, carvedilol; Col I, type I collagen; FN, fibronectin; p, phosphorylated; TGF $\beta 1$, transforming growth factor $\beta 1$.

were not described (25). In the present study, carvedilol not only improved the hepatotoxicity indicators, but also improved hepatic architectural distortion and liver fibrosis in cirrhotic rats. Structural changes are important factors in the development of IHVR, which is the determinant factor of PHT (6). Previous studies have demonstrated that improvement of liver fibrosis can reduce portal pressure $(17,26)$. Therefore, the antifibrogenic effect of carvedilol may be involved in reducing portal pressure. HSCs are the target of the antifibrogenic therapy for hepatic fibrosis $(27,28)$. A number of agents targeting activated HSCs have demonstrated their antifibrogenic effect in animal models (29-32). Preventing HSC activation, proliferation, migration and collagen synthesis are major objectives in the treatment of liver fibrosis $(27,28,33)$. The present study demonstrated that carvedilol may exert antifibrogenic effects in cirrhotic rats by inhibiting the proliferation, migration, activation and collagen synthesis in HSCs.

HSCs are the resident perisinusoidal cells in the space of Disse, and are the central effector in hepatic fibrosis (7). In response to liver injury, HSCs are activated and undergo phenotypic transformation to a myofibroblastic phenotype characterized by proliferation, migration to sites of injury, increased production of profibrogenic cytokines, and elevated accumulation of ECM components including Col I and FN $(10,34)$. Among the profibrogenic cytokines, TGF $\beta 1$ is the most potent profibrogenic cytokine; TGF $\beta 1$ promotes the accumulation of ECM proteins in the progression of liver fibrosis (12). TGF $\beta 1$ was used in the present study to stimulate LX-2 cells to explore the mechanisms underlying the antifibrogenic effect of carvedilol. The results demonstrated that TGF $\beta 1$ upregulated the collagen synthesis in LX-2 cells, which was consistent with previous studies $(12,35)$.

The present study results demonstrated that pretreatment with carvedilol decreased TGF $\beta 1$-induced collagen synthesis in LX-2 cells. TGF $\beta 1$ activates SMAD-dependent and SMAD-independent pathways, including PI3K/AKT and MAPK pathways such as ERK (36-38). The TGF $\beta /$ SMAD pathway is a major signaling pathway in the liver in both normal and pathological conditions $(36,37)$. In the SMAD-dependent pathway, members of the SMAD family transmit signals from the cell surface into the nucleus. Following stimulation by
TGF $\beta 1$, SMAD 2 and SMAD3 are phosphorylated and form a heterotrimeric complex with SMAD4 $(36,37)$. This complex translocates into the nucleus and regulates the expression of target genes $(36,37)$. The results of the present study revealed that TGF $\beta 1$ activated the SMAD-dependent pathway in LX-2 cells, and pretreatment with carvedilol decreased the TGF 31 -induced phosphorylation of SMAD2 and SMAD3. As a potent and selective inhibitor of SMAD3, SIS3 blocked the upregulation of collagen synthesis in TGF $\beta 1$-stimulated LX-2 cells. These results demonstrated that carvedilol may reduce the TGF $\beta 1$-induced increase of collagen synthesis in LX-2 cells by inhibiting the TGF $\beta 1 /$ SMAD pathway.

In conclusion, the present study demonstrated that carvedilol may improve liver cirrhosis in rats by inhibiting HSCs proliferation, invasion, activation and collagen synthesis. Furthermore, carvedilol may inhibit collagen synthesis in HSCs by suppressing the TGF $\beta 1 /$ SMAD pathway. Therefore, the application of carvedilol in chronic liver diseases may be extended beyond the pharmacological treatment of PHT in patients with decompensated cirrhosis, and carvedilol may be applied in the treatment of early-stage liver cirrhosis.

\section{Acknowledgements}

Not applicable.

\section{Funding}

The present study was supported by The National Nature Science Foundation of China (grant no. 81370590).

\section{Availability of data and materials}

All data generated or analyzed during this study are included in this published article.

\section{Authors' contributions}

LL performed experiments and wrote the manuscript. GL and GW performed the experiments. DM and ZL maintained the animals and established the liver cirrhosis animal model. CZ 
designed the study. All authors read and approved the final manuscript.

\section{Ethics approval and consent to participate}

All experimental procedures and protocols were approved by the Animal Medical Ethics Committee of Shandong Provincial Hospital affiliated to Shandong University (Jinan, China).

\section{Patient consent for publication}

Not applicable.

\section{Competing interests}

The authors declare that they have no competing interests.

\section{References}

1. Fernandez M: Molecular pathophysiology of portal hypertension. Hepatology 61: 1406-1415, 2015

2. Bosch J, Groszmann RJ and Shah VH: Evolution in the understanding of the pathophysiological basis of portal hypertension: How changes in paradigm are leading to successful new treatments. J Hepatol 62: S121-S130, 2015.

3. Reiberger T, Ferlitsch A, Payer BA, Pinter M, Homoncik M and Peck-Radosavljevic M; Vienna Hepatic Hemodynamic Lab: Non-selective $\beta$-blockers improve the correlation of liver stiffness and portal pressure in advanced cirrhosis. J Gastroenterol 47: 561-568, 2012.

4. Bosch J, Abraldes JG, Fernández M and García-Pagán JC: Hepatic endothelial dysfunction and abnormal angiogenesis: New targets in the treatment of portal hypertension. J Hepatol 53: 558-567, 2010.

5. Iwakiri Y and Groszmann RJ: Vascular endothelial dysfunction in cirrhosis. J Hepatol 46: 927-934, 2007.

6. Sanyal AJ, Bosch J, Blei A and Arroyo V: Portal hypertension and its complications. Gastroenterology 134: 1715-1728, 2008.

7. Lee UE and Friedman SL: Mechanisms of hepatic fibrogenesis. Best Pract Res Clin Gastroenterol 25: 195-206, 2011.

8. Senoo H, Yoshikawa K, Morii M, Miura M, Imai K and Mezaki Y: Hepatic stellate cell (vitamin A-storing cell) and its relative-past, present and future. Cell Biol Int 34: 1247-1272, 2010.

9. Xu L, Hui AY, Albanis E, Arthur MJ, O'Byrne SM, Blaner WS, Mukherjee P, Friedman SL and Eng FJ: Human hepatic stellate cell lines, LX-1 and LX-2: New tools for analysis of hepatic fibrosis. Gut 54: 142-151, 2005.

10. Reynaert H, Thompson MG, Thomas T and Geerts A: Hepatic stellate cells: Role in microcirculation and pathophysiology of portal hypertension. Gut 50: 571-581, 2002.

11. Friedman SL: Molecular regulation of hepatic fibrosis, an integrated cellular response to tissue injury. J Biol Chem 275: 2247-2250, 2000.

12. Bissell DM, Roulot D and George J: Transforming growth factor beta and the liver. Hepatology 34: 859-867, 2001.

13. de Franchis R; Baveno VI Faculty: Expanding consensus in portal hypertension: Report of the Baveno VI Consensus Workshop: Stratifying risk and individualizing care for portal hypertension. J Hepatol 63: 743-752, 2015.

14. Arozal W, Sari FR, Watanabe K, Arumugam S, Veeraveedu PT, Ma M, Thandavarayan RA, Sukumaran V, Lakshmanan AP, Kobayashi Y, et al: Carvedilol-afforded protection against daunorubicin-induced cardiomyopathic rats in vivo: Effects on cardiac fibrosis and hypertrophy. ISRN Pharmacol 2011: 430549, 2011.

15. Wong VY, Laping NJ, Nelson AH, Contino LC, Olson BA, Gygielko E, Campbell WG Jr, Barone F and Brooks DP: Renoprotective effects of carvedilol in hypertensive-stroke prone rats may involve inhibition of TGF beta expression. Br $\mathbf{J}$ Pharmacol 134: 977-984, 2001.

16. El-Demerdash E, Abdel-Sattar SA, El-Bakly WM and Mohamed EA: Antifibrotic effects of carvedilol and impact of liver fibrosis on carvedilol pharmacokinetics in a rat model. Eur J Drug Metab Pharmacokinet 42: 767-779, 2017.
17. Krogsgaard K, Gluud C, Henriksen JH and Christoffersen P: Correlation between liver morphology and portal pressure in alcoholic liver disease. Hepatology 4: 699-703, 1984.

18. Starkel P and Leclercq IA: Animal models for the study of hepatic fibrosis. Best Pract Res Clin Gastroenterol 25: 319-333, 2011.

19. Andrade GB, Montes GS, Conceição GM and Saldiva PH: Use of the Picrosirius-polarization method to age fibrotic lesions in the hepatic granulomas produced in experimental murine schistosomiasis. Ann Trop Med Parasitol 93: 265-272, 1999.

20. Jinnin M, Ihn H and Tamaki K: Characterization of SIS3, a novel specific inhibitor of Smad3, and its effect on transforming growth factor-beta1-induced extracellular matrix expression. Mol Pharmacol 69: 597-607, 2006.

21. Tripathi D and Hayes PC: Beta-blockers in portal hypertension: New developments and controversies. Liver Int 34: 655-667, 2014.

22. Carlson W and Oberg K: Clinical pharmacology of carvedilol. J Cardiovasc Pharmacol Ther 4: 205-218, 1999.

23. Ding Q, Tian XG, Li Y, Wang QZ and Zhang CQ: Carvedilol may attenuate liver cirrhosis by inhibiting angiogenesis through the VEGF-Src-ERK signaling pathway. World J Gastroenterol 21: 9566-9576, 2015.

24. Barone FC, Campbell WG Jr, Nelson AH and Feuerstein GZ: Carvedilol prevents severe hypertensive cardiomyopathy and remodeling. J Hypertens 16: 871-884, 1998.

25. Hamdy $\mathrm{N}$ and El-Demerdash E: New therapeutic aspect for carvedilol: Antifibrotic effects of carvedilol in chronic carbon tetrachloride-induced liver damage. Toxicol Appl Pharmacol 261: 292-299, 2012.

26. Di Pascoli M, Divi M, Rodriguez-Vilarrupla A, Rosado E, Gracia-Sancho J, Vilaseca M, Bosch J and García-Pagán JC: Resveratrol improves intrahepatic endothelial dysfunction and reduces hepatic fibrosis and portal pressure in cirrhotic rats. J Hepatol 58: 904-910, 2013.

27. Wu J and Zern MA: Hepatic stellate cells: A target for the treatment of liver fibrosis. J Gastroenterol 35: 665-672, 2000.

28. Schuppan D and Popov Y: Hepatic fibrosis: From bench to bedside. J Gastroenterol Hepatol 17 (Suppl 3): S300-S305, 2002.

29. Kaji K, Yoshiji H, Ikenaka Y, Noguchi R, Aihara Y, Douhara A, Moriya K, Kawaratani H, Shirai Y, Yoshii J, et al: Dipeptidyl peptidase-4 inhibitor attenuates hepatic fibrosis via suppression of activated hepatic stellate cell in rats. J Gastroenterol 49: 481-491, 2014.

30. Li J, Li X, Xu W, Wang S, Hu Z, Zhang Q, Deng X, Wang J, Zhang J and Guo C: Antifibrotic effects of luteolin on hepatic stellate cells and liver fibrosis by targeting AKT/mTOR/p70S6K and TGF $3 /$ Smad signalling pathways. Liver Int 35: 1222-1233, 2015.

31. Zhu J, Wu J, Frizell E, Liu SL, Bashey R, Rubin R, Norton P and Zern MA: Rapamycin inhibits hepatic stellate cell proliferation in vitro and limits fibrogenesis in an in vivo model of liver fibrosis. Gastroenterology 117: 1198-1204, 1999.

32. Son G, Hines IN, Lindquist J, Schrum LW and Rippe RA: Inhibition of phosphatidylinositol 3-kinase signaling in hepatic stellate cells blocks the progression of hepatic fibrosis. Hepatology 50: 1512-1523, 2009.

33. Trautwein C, Friedman SL, Schuppan D and Pinzani M: Hepatic fibrosis: Concept to treatment. J Hepatol 62: S15-S24, 2015.

34. Greuter T and Shah VH: Hepatic sinusoids in liver injury, inflammation, and fibrosis: New pathophysiological insights. J Gastroenterol 51: 511-519, 2016.

35. Kajdaniuk D, Marek B, Borgiel-Marek H and Kos-Kudła B: Transforming growth factor $\beta 1$ (TGF $\beta 1)$ in physiology and pathology. Endokrynol Pol 64: 384-396, 2013.

36. Inagaki $Y$ and Okazaki I: Emerging insights into transforming growth factor beta Smad signal in hepatic fibrogenesis. Gut 56: 284-292, 2007.

37. Derynck R and Zhang YE: Smad-dependent and Smadindependent pathways in TGF-beta family signalling. Nature 425: 577-584, 2003.

38. Bakin AV, Tomlinson AK, Bhowmick NA, Moses HL and Arteaga CL: Phosphatidylinositol 3-kinase function is required for transforming growth factor beta-mediated epithelial to mesenchymal transition and cell migration. J Biol Chem 275: 36803-36810, 2000 .

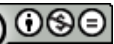

This work is licensed under a Creative Commons Attribution-NonCommercial-NoDerivatives 4.0 International (CC BY-NC-ND 4.0) License. 